FORMATION Formation emploi

Revue française de sciences sociales

102 | avril-juin 2008

Pêle-mêle

\title{
Esquisse d'une profession consultante : les intermédiaires du marché du travail en Wallonie
}

Portrait of a consultants' occupation. Mediators on the Walloon labour market Bosquejo de una profesión de consultor. Los intermediarios del mercado de trabajo en Wallonie

Porträt einer beratenden Profession: Wallonische Arbeitsmarkt-Vermittler

Jean-François Orianne et Christian Maroy

\section{(Q) OpenEdition}

Journals

Édition électronique

URL : http://journals.openedition.org/formationemploi/2221

DOI : $10.4000 /$ formationemploi.2221

ISSN : 2107-0946

Éditeur

La Documentation française

Édition imprimée

Date de publication : 1 juin 2008

Pagination : 21-39

ISSN : 0759-6340

Référence électronique

Jean-François Orianne et Christian Maroy, «Esquisse d'une profession consultante : les intermédiaires du marché du travail en Wallonie », Formation emploi [En ligne], 102 I avril-juin 2008, mis en ligne le 01 juin 2009, consulté le 30 octobre 2020. URL : http://journals.openedition.org/formationemploi/2221 ; DOI : https://doi.org/10.4000/formationemploi.2221 


\title{
étrangers
}

Pays-et

\section{Esquisse d'une profession consultante: les intermédiaires du marché du travail en Wallonie ${ }^{1}$}

par Jean-François Orianne et Christian Maroy*

\begin{abstract}
De nouveaux groupes professionnels diagnostiquent, chez les demandeurs d'emploi, des troubles de l'employabilité afin d'amener ces derniers à " travailler sur eux-mêmes », de les faire tendre vers une certaine autonomie.
\end{abstract}

Les politiques dites « actives » d'emploi² se développent en Belgique comme partout en Europe (Barbier et Gautié, 1998; Maroy, 2000). Les analyses critiques ont surtout pointé les dangers que ces politiques génèrent, en soulignant, d'une part, les risques d'une

\footnotetext{
${ }^{1}$ Cet article présente les principaux résultats d'une recherche empirique, menée au GIRSEF (Groupe interfacultaire de recherche sur les systèmes d'éducation et de formation), qui interroge les modalités de mise en œuvre des politiques actives d'emploi en Belgique : Orianne J.-F., Maroy C., Moulaert T., Vandenberghe V., Waltenberg F. (2004), Mises en æuvre locales des formules d'activation des politiques d'emploi, Academia Press, Gent.

Cette recherche a bénéficié d'un financement de la Politique scientifique fédérale dans le cadre du Programme de recherche pluriannuel concernant les problèmes actuels en matière de cohésion sociale. Le travail de terrain s'est déroulé en Région wallonne de Belgique, entre mars 2001 et juin 2003, principalement dans les sous-régions de Liège (contexte urbain) et d'Arlon-Luxembourg (contexte rural). Notre matériau se compose d'une cinquantaine d'entretiens approfondis d'agents d'insertion, de nombreuses observations (enregistrées) d'interactions entre agents et demandeurs d'emploi, de compte-rendu de suivi d'agents au quotidien. ${ }^{2}$ Les politiques de l'emploi, sous l'influence des institutions européennes, se sont progressivement transformées en politiques dites d' « employabilité », abandonnant de la sorte leur vocation régulatrice et créatrice d'emplois : à la fonction keynésienne de soutien à la croissance se substitue une autre fonction, davantage centrée.../...
}

\begin{abstract}
* Jean-François Orianne est chargé de cours à l'Institut des sciences humaines et sociales de l'université de Liège, professeur à l'ICHEC (Institut catholique des hautes études commerciales) et chercheur associé à l'IDHE (Institutions et dynamiques historiques de l'économie - École normale supérieure de Cachan) et à l'Observatoire social européen. Sa thèse de doctorat, réalisée au GIRSEF Groupe interfacultaire de recherche sur les systèmes d'éducation et de formation $/ \mathrm{UCL}$ - univesité catholique de Louvain), traite de la mise en œeuvre des politiques actives d'emploi. II mène diverses recherches dans le champ des politiques d'emploi et de formation professionnelle.
\end{abstract}

\begin{abstract}
.../... sur les capacités et la responsabilité individuelles (Orianne et Conter, 2007). Dans ce contexte, l'activation des politiques d'emploi signifie conjointement: 1) l'activation des dépenses de l'État afin d'augmenter l'efficacité des dépenses publiques (l'intervention publique est conditionnée); 2) l'activation du bénéficiaire, en le responsabilisant dans sa démarche d'insertion ou d'intégration (individualisation); 3) l'activation des professionnels chargés de mettre en œuvre les politiques sociales (territorialisation) (Orianne et al., 2004, p. 110).
\end{abstract}


Ses travaux récents, réalisés dans le cadre du réseau CAPRIGHT, portent sur les services publics d'emploi en Europe et sur le dialogue social européen. Il a publié récemment : (avec Jean De Munck), "Droits sociaux et mondes possibles. L'exemple du droit au congé parental », Raisons pratiques, $n^{\circ} 18$, Éditions de l'EHESS, Paris, 2008, pp. 263-280 ; (avec Marie Verhoeven et Vincent Dupriez), "Vers des politiques d'éducation "capacitantes" », Formation Emploi, n 98, 2007, pp. 93-108.

Christian Maroy est directeur du GIRSEF et professeur de sociologie à l'UCL. II dirige diverses recherches dans le champ des politiques d'éducation et de formation. Ses travaux récents, réalisés dans le cadre du réseau REGULEDUC, portent sur les régulations des systèmes éducatifs en Europe. II a publié récemment: lavec Lise Demailly), "Les régulations intermédiaires des systèmes éducatifs en Europe: quelles convergences ? », Recherches sociologiques, 2004/2, pp. 5-23. École, régulation et marché. Une comparaison de six espaces scolaires locaux en Europe, PUF, Paris, 2006.

marchandisation, et d'une privatisation accrue des services de l'emploi (Gautié, 2003 ; Raveaud, 2004), en dénonçant, d'autre part, les possibles dérives autoritaires (Alaluf, 2004 ; Hamzaoui, 2005). En outre, du point de vue des syndicats et du monde associatif, l'État social actif, comme on le qualifie en Belgique, serait essentiellement un État coercitif, contrôleur, se livrant à diverses formes de "chasse aux chômeurs » (Faniel, 2005). Sans nier que ces tendances sont présentes, il nous semble utile de souligner qu'elles cachent peut-être une tendance plus souterraine: le traitement clinique des chômeurs (Orianne, 2005b). Nous entendons par clinique la méthode qui consiste à poser un diagnostic à partir de l'observation directe de symptômes.

En effet, dans le cadre de dispositifs contraignants d'« accompagnement» des demandeurs d'emploi ${ }^{3}$, le travail d'insertion consiste principalement, et de plus en plus, à mettre l'individu au travail... sur luimême. Cette orientation nous semble liée à l'émergence de divers groupes professionnels qui tendent à se construire comme une profession consultante

\footnotetext{
${ }^{3}$ Précision que ces dispositifs sont obligatoires pour tout demandeur d'emploi inscrit dans un office régional (par exemple, le FOREM en Wallonie) sous peine de sanction administrative de l'Office national de l'emploi (ONEM).
}

(Freidson, 1984), disposant d'une expertise et d'un monopole très particuliers. Leur légitimité professionnelle, vis-à-vis du public, repose essentiellement sur leur capacité à diagnostiquer des «troubles de l'employabilité» (par exemple, le manque de confiance en soi, la maîtrise insuffisante des techniques de recherche d'emploi, l'absence de projet professionnel, le manque d'expérience professionnelle, divers « troubles » de l'orientation professionnelle ou de la motivation, les « problèmes » familiaux, etc.). Avec la collaboration active des «patients», il s'agit de repérer des atouts et des déficiences en termes de compétences, et d'orienter les demandeurs d'emploi vers divers spécialistes de l'employabilité qui pourront envisager un «traitement» des troubles ainsi identifiés. L'autonomie professionnelle de ces agents, vis-à-vis de l'État, implique également, comme nous le verrons, une déconnexion entre le travail sur l'employabilité et la mise à l'emploi : «on n'est pas là pour parler en termes de solution (...) le sens de notre travail, ce n'est pas forcément que les gens travaillent, (...) ce n'est pas la mise à l'emploi », ne cessent-ils de répéter.

Notre contribution souligne le rôle des intermédiaires publics du marché du travail ${ }^{4}$ dans l'orientation des politiques actives d'emploi en Région wallonne (Belgique) : c'est par leurs choix éthiques et techniques que se construit l'orientation clinique du traitement des chômeurs. C'est eux qui tendent à faire de l'autonomie de la personne en recherche d'emploi le sens dominant du travail d'insertion. L'autonomie constitue ainsi une traduction professionnelle du concept d'employabilité, dans une orientation « humaniste », une sorte de « troisième voie » à michemin entre le tri du marché (l'employabilité marchande) et la sanction de l'État (le contrôle des chômeurs). Appuyé sur la sociologie interactionniste des professions, cet article vise ainsi à contribuer à une sociologie de l'action publique (Duran, 1999 ; Simonin, 1995), qui se propose de substituer à une analyse classique des politiques publiques centrée sur les mesures, une analyse de l'action publique centrée sur les agents locaux qui mettent en œuvre les politiques, notamment dans le domaine de l'emploi.

\footnotetext{
${ }^{4}$ Appelés également intermédiaires des politiques publiques de l'emploi (Gélot et Nivolle, 2000).
} 
Notre propos s'appuie sur une recherche, menée au GIRSEF (UCL), qui interroge les modalités de mise en œuvre des politiques actives d'emploi (Orianne et al., 2004) $)^{5}$, en particulier de la politique du Parcours d'insertion ${ }^{6}$. Cette recherche, de type ethnographique

\footnotetext{
${ }^{5}$ Cette recherche a bénéficié d'un financement de la Politique scientifique fédérale dans le cadre du Programme de recherche pluri-annuel concernant les problèmes actuels en matière de cohésion sociale.

${ }^{6}$ Le Parcours d'insertion est une politique européenne de l'emploi menée depuis le milieu des années 90 , au nom d'une meilleure.../...
}

(voir encadré 1), a porté sur trois groupes professionnels issus du service public d'emploi, du secteur

.../... intégration et coordination des multiples actions destinées aux personnes en recherche d'emploi (Maroy et Van Haeperen, 2005). Le Parcours d'insertion est défini comme un dispositif global d'accompagnement des demandeurs d'emploi vers l'emploi : il vise le développement de l'employabilité des chômeurs (capacité d'insertion socioprofessionnelle, « chances » d'accéder à un emploi durable et de qualité). En Belgique, cette politique européenne se traduit par des accords de coopération entre l'État fédéral et les Régions. En Région wallonne, la mise en œuvre du Parcours d'insertion date de 1997 et, depuis 2004, cette politique s'inscrit dans un cadre légal : le décret du Gouvernement wallon du 01/04/2004 relatif au dispositif intégré d'insertion socioprofessionnelle.

\section{Encadré 1}

\section{Le travail de terrain}

Notre travail de terrain s'est déroulé en Région wallonne (Belgique) entre mars 2001 et juin 2003, principalement dans les sous-régions de Liège (contexte urbain) et d'Arlon-Luxembourg (contexte rural). Nous y avons suivi, dans la quotidienneté de leur travail, une cinquantaine d'agents issus de diverses organisations du "dispositif intégré d'insertion ". Dans chaque sous-région, nous avons privilégié trois entrées organisationnelles contrastées: le Service public d'emploi (FOREM), des structures multi-partenariales (Carrefours Formation) et des associations œuvrant dans le champ de l'insertion (Entreprises de formation par le travail). Nous nous sommes principalement centrés sur trois groupes professionnels : les conseillers en accompagnement professionnels du FOREM, les conseillers en formation des Carrefours Formation et les travailleurs sociaux des Entreprises de formation par le travail.

La recherche a commencé par une phase exploratoire d'analyse de documents "institutionnels 》 relatifs aux politiques actives d'emploi, couplée à une première série d'entretiens. La documentation institutionnelle comprend toute une série de lois (ou décrets), règlements, conventions-cadres, chartes de partenariat... La première série d'entretiens regroupe d'une part les quelques réunions de travail avec les évaluateurs du Parcours d'insertion en Région wallonne, et d'autre part, quelques entretiens exploratoires auprès de responsables de diverses organisations spécialisées dans le traitement des chômeurs $(n=14)$. Lors de la phase principale d'enquête, nous avons réalisé une cinquantaine d'entretiens d'agents ( $n=49$ ) et une trentaine d'entretiens d'usagers ( $n=32)$. Nous avons, en outre, observé de nombreuses interactions entre agents et usagers, dont une trentaine ont pu être intégralement enregistrées $(n=30)$. Nous avons également observé de nombreuses interactions entre agents, dans la quotidienneté de leur travail et dans le cadre particulier de réunions d'équipes $(n=25)$ ou de réunions de travail chez des "opérateurs partenaires 》 (professionnels issus d'autres organisations du dispositif intégré d'insertion avec qui ils collaborent; $n=16$ ). Nous avons enfin procédé à l'analyse des outils de travail de ces agents: outils de communication (interne et externe), outils pédagogiques, outils de traitement et d'analyse de données, outils de traduction de dispositifs réglementaires...

Une dernière phase de "clôture » ou de retour sur le terrain correspond aux divers entretiens complémentaires et contacts ponctuels pendant la phase d'analyse du matériau, jusqu'à la phase finale de rédaction du rapport. Elle comprend également la présentation des résultats de l'étude dans chaque équipe ( $n=6$ ) et l'organisation d'un séminaire au GIRSEF (Groupe inter-facultaire de recherche sur les systèmes d'éducation et de formation - UCL) réunissant chercheurs et acteurs de terrain. 
associatif de l'insertion, et de structures partenariales : 1) les conseillers en accompagnement professionnel $\mathrm{du} \mathrm{FOREM}^{7}$, 2) les conseillers en formation de Carrefour Formation, 3) les travailleurs sociaux en Entreprises de Formation par le Travail ( $c f$. tableau 1).

Nous présenterons d'abord de façon descriptive le travail de ces trois groupes professionnels, en particulier les modalités de construction (ou de production de diagnostics) de « troubles de l'employabilité » à laquelle procèdent les trois types de travailleurs investigués. À la lumière de la sociologie interactionniste des professions, nous montrerons ensuite que leur action peut s'interpréter comme typique du travail d'une profession consultante, qui établit les troubles de l'employabilité et permet au demandeur d'emploi, dans un contexte de chômage de masse persistant, de se conduire en «malade», porteur de ces troubles, et (co-)responsable de son « traitement». Nous soulignerons enfin le fait que leur action présente des variations qui peuvent s'interpréter à l'aune d'une typologie des conventions de compétences (ou «façons » de les évaluer) empruntée aux travaux conventionnalistes ${ }^{8}$ (Eymard-Duvernay et Marchal, 1997).

\section{LE CONSEILLER EN ACCOMPAGNEMENT PROFESSIONNEL : GÉNÉRALISTE DE L’EMPLOYABILITÉ}

Le conseiller en accompagnement professionnel (CAP), appartenant au service « Conseil » du Service

\footnotetext{
${ }^{7}$ Le FOREM est l'Office de la formation professionnelle et de l'emploi ; il constitue le Service public d'emploi en Région wallonne. Rappelons que la Belgique est un état fédéral ; la gestion des allocations de chômage reste une compétence fédérale au sein de l'Office national de l'emploi (ONEM) alors que la « gestion du marché du travail » (mesures relatives à la recherche d'emploi, à la rencontre offre/demande d'emploi, à la formation professionnelle ou à l'accompagnement des demandeurs d'emploi) relève plus spécifiquement du FOREM en ce qui concerne la Région wallonne.

${ }^{8}$ Il s'agit des travaux issus de l'école (française) de l'Économie des conventions appliqués à l'analyse des intermédiaires du marché du travail et des politiques d'emploi (Simonin, 1995 ; Bessy et Eymard-Duvernay, 1997 ; Eymard-Duvernay et Marchal, 1997 ; Meyer, 1998 ; Gélot et Nivolle, 2000 ; Bessy et al., 2001 ; etc.).
}

public d'emploi en Région wallonne (FOREM), est un métier qui émerge de lourdes transformations organisationnelles et institutionnelles. Celles-ci ont été générées, d'une part, par le développement de politiques actives d'emploi - en particulier la mise en œuvre de la politique européenne du Parcours d'insertion - et d'autre part, par la ratification de la convention 181 de l'OIT (Organisation internationale du travail) qui autorise les opérateurs privés à offrir des services en matière de placement des travailleurs. La redéfinition à l'échelle européenne de l'objectif des politiques d'emploi en termes d'employabilité, ainsi qu'une nouvelle situation de concurrence entre les intermédiaires publics et privés du marché du travail sont deux éléments contextuels qui ont directement contribué à mettre un terme au « règne» du placeur public, - ce groupe professionnel qui vit le jour en 1935 lors de la création de l'Office national du placement et du chômage. Le CAP est un descendant direct du placeur, mais il ne place plus ; il apparaît comme la concrétisation la plus aboutie, à ce jour, d'une nouvelle logique d'accompagnement des demandeurs d'emploi, qui s'est progressivement substituée à l'ancienne fonction de placement des chômeurs.

Le Gouvernement wallon accorde à ce nouveau groupe professionnel une licence ${ }^{9}$ (au sens de Hughes, 1996: l'autorisation légale d'exercer) exclusive pour exercer son activité : ainsi, il le charge spécifiquement de suivre les bénéficiaires tout au long de leur parcours d'insertion contractualisé (ou contrat crédit insertion) ${ }^{10}$. Le CAP est défini comme le référent unique chargé d'accueillir, d'informer, d'accompagner, de " coacher» de manière «proactive» et individualisée les demandeurs

\footnotetext{
${ }^{9}$ «On peut dire qu'un métier existe lorsqu'un groupe de gens $s$ 'est fait reconnaître la licence exclusive d'exercer certaines activités en échange d'argent, de biens ou de services. Ceux qui disposent de cette licence, s'ils ont le sens de la solidarité et de leur propre position, revendiqueront un mandat pour définir les comportements que devraient adopter les autres personnes à l'égard de tout ce qui touche à leur travail. (...) Licence et mandat constituent les manifestations premières de la division morale du travail, c'est-à-dire des processus de répartition des différentes fonctions morales entre les membres de la société, individus ou catégories d'individus » (Hughes, 1996, pp. 99-100).

${ }^{10}$ Gouvernement wallon, décret du 01/04/2004 relatif au dispositif intégré d'insertion socioprofessionnelle, article 5, Namur.
} 
Tableau 1

Trois groupes d'intermédiaires du marché du travail en Région wallonne

\begin{tabular}{|c|c|c|c|}
\hline & $\begin{array}{l}\text { Le conseiller en } \\
\text { accompagnement professionnel } \\
\text { (CAP) du FOREM }\end{array}$ & $\begin{array}{l}\text { Le conseiller en formation } \\
\text { de Carrefour Formation }\end{array}$ & $\begin{array}{l}\text { Le formateur en Entreprise } \\
\text { de Formation par le Travail }\end{array}$ \\
\hline Origine & Placeur public (1935) & $\begin{array}{l}\text { Instructeur en gestion de projets } \\
\text { professionnels (FOREM, 1995) }\end{array}$ & $\begin{array}{c}\text { Formateur en Entreprise } \\
\text { d'Apprentissage Professionnel } \\
\text { (1987) }\end{array}$ \\
\hline Fonction & Généraliste de l'employabilité & $\begin{array}{c}\text { Spécialiste de l'employabilité : } \\
\text { information et conseil } \\
\text { en formation }\end{array}$ & $\begin{array}{l}\text { Généraliste } \\
\text { pour publics spécialisés }\end{array}$ \\
\hline $\begin{array}{l}\text { Type } \\
\text { d'organisation }\end{array}$ & $\begin{array}{l}\text { Service public d'emploi } \\
\text { en Région wallonne }\end{array}$ & $\begin{array}{c}\text { Structures partenariales } \\
\text { (composées de cinq opérateurs } \\
\text { de formation) }\end{array}$ & $\begin{array}{l}\text { Secteur associatif de l'insertion } \\
\text { (ASBL) }\end{array}$ \\
\hline Licence & $\begin{array}{c}\text { Décret du } 01 / 04 / 2004 \text { relatif } \\
\text { au dispositif intégré d'insertion } \\
\text { socioprofessionnelle (art. 5) }\end{array}$ & $\begin{array}{l}\text { Convention Cadre de } 1998 . \\
\text { Décret du } 01 \text { /04/2004 relatif } \\
\text { au dispositif intégré d'insertion } \\
\text { socioprofessionnelle (art. 9) }\end{array}$ & $\begin{array}{l}\text { Décret du } 01 / 04 / 2004 \\
\text { relatif à l'agrément } \\
\text { et au subventionnement } \\
\text { des EFT/OISP (*) }\end{array}$ \\
\hline Mandat & $\begin{array}{c}\text { Profil de fonction CAP (FOREM: } \\
\text { service public d'emploi). } \\
\text { Campagne de communication } \\
\text { du FOREM }\end{array}$ & $\begin{array}{c}\text { Charte de l'animation } \\
\text { pédagogique (1997). } \\
\text { Rapports annuels du Comité } \\
\text { d'Accompagnement } \\
\text { des Carrefour Formation }\end{array}$ & $\begin{array}{l}\text { Rapports de l'Interfédération } \\
\text { et des cinq fédérations } \\
\text { d'EFT/OISP. } \\
\text { Rapports d'activité des EFT }\end{array}$ \\
\hline Statut & Contractuel & $\begin{array}{c}\text { Contractuel } \\
\text { (variable en fonction } \\
\text { de l'opérateur partenaire) }\end{array}$ & $\begin{array}{c}\text { Agent contractuel } \\
\text { subventionné : chômeurs mis } \\
\text { au travail par le nouveau } \\
\text { programme de résorption } \\
\text { du chômage (PRC) }\end{array}$ \\
\hline Rémunération & $\begin{array}{c}\text { Échelle barémique : } \\
\text { B3 - 1547,47 euros mensuel } \\
\text { brut indexé }\end{array}$ & $\begin{array}{l}\text { Variable en fonction } \\
\text { de l'opérateur partenaire: } \\
\text { salaire mensuel brut } \\
\text { entre } 1300 \text { et } 2000 \text { euros }\end{array}$ & $\begin{array}{l}\text { Salaire mensuel brut: } \\
\text { entre } 1300 \text { et } 2300 \text { euros } \\
\text { (cf. CCT n } 64812 \text { et décret } \\
\text { du } 25 / 04 / 2002 \text { l }\end{array}$ \\
\hline $\begin{array}{l}\text { Niveau de } \\
\text { diplôme }\end{array}$ & $b a c+3$ & $\begin{array}{c}\text { Variable en fonction } \\
\text { de l'opérateur partenaire } \\
\text { bac, bac }+3, \text { bac }+5\end{array}$ & $\begin{array}{l}\text { 1) soit des hommes de métier } \\
\text { « reconvertis » (peintres } \\
\text { en bâtiment, maçons, etc.) } \\
\text { 2) soit bac }+3 \text { (orientation } \\
\text { travail social) }\end{array}$ \\
\hline
\end{tabular}

Note de lecture : le mandat, comme nous le préciserons plus loin, constitue une traduction professionnelle de l'autorisation légale d'exercer (la licence) : en clair, la reformulation par les professionnels (au travers de leurs organisations) des missions légales qui leur sont confiées par l'État (ici, le Gouvernement wallon). Pour chaque groupe étudié, une pluralité de documents, de natures très diverses, rend compte de cette activité incessante d'opérationnalisation de leur licence (générale et abstraite). Au FOREM, on s'efforce de traduire les missions officielles en profils de fonction (la liste des compétences attendues d'un conseiller) ou encore en offre de services (la liste des actions proposées par les différentes lignes de services du FOREM). Chez Carrefour Formation, le mandat prend la forme d'une charte - fruit d'un travail collectif - par laquelle les différents opérateurs partenaires s'engagent contractuellement à poursuivre des objectifs et intérêts communs (mettre en œuvre les deux missions spécifiques d'information et de conseil) dans un même espace de travail et à respecter un même code déontologique (valeurs communes de travail : écoute structurée, respect de l'usager, empathie, etc.). Il prend enfin la forme de rapports officiels, en EFT comme chez Carrefour Formation, qui précisent les contours et le sens de l'intervention.

(*) OISP : Organismes d'insertion socioprofessionnelle; EFT : entreprises de formation par le travail. 
d'emploi ${ }^{11}$. Sur la base de cette licence officielle, une organisation professionnelle se constitue au sein de l'administration centrale du FOREM, et œuvre à la traduction professionnelle de l'autorisation légale d'exercer, à l'élaboration d'un mandat (Hughes, op. cit. ; Dubar et Tripier, 1998, p. 104). Il ressort de cette définition organisationnelle du métier deux éléments essentiels, étroitement liés : la fonction de diagnostic et l'image d'un généraliste qui renvoie à des spécialistes. Sur un plan identitaire, le CAP se définit comme un " généraliste de l'employabilité », dont la tâche principale est d'accompagner des individus qui travaillent leur employabilité. En pratique, ce travail d'accompagnement nécessite de prendre en compte différents troubles de l'employabilité, de les analyser, d'en dresser la symptomatologie, de les diagnostiquer dans une approche globale de l'individu, et de renvoyer les individus à des spécialistes compétents :

«Est-ce que ça se dessine clairement, pour toi, la spécificité du CAP dans l'ensemble de cette structure? C'est un peu le médecin généraliste qui va renvoyer à des spécialistes. Tu sais, c'est vraiment rattaché au corps médical; je trouve que l'image n'est pas si mauvaise que ça. (...) Donc on va faire tout un travail de visualisation, de relevé de tout... enfin si on parle le langage médical -, le relevé de toute la symptomatologie qui nous permet d'établir à terme un diagnostic. » (Conseiller en Accompagnement Professionnel, FOREM de Libramont)

Les modes opératoires (méthode de travail, contrôle des opérations, ...) des CAP sont principalement définis par des règlements, plus exactement par des " chaînes télescopiques" de traduction de règlements, autrement dit, des traductions de traduction (de traduction, etc.) des énoncés des politiques actives d'emploi. Ces modes opératoires s'acquièrent dans la pratique et dans le cadre de formations programmées par l'administration centrale du FOREM. Un vade mecum structure le travail d'accompagnement en différentes étapes : l'accueil, le pré-repérage, l'analyse approfondie de la demande,

${ }^{11}$ Avant-projet de décret relatif au dispositif intégré d'insertion socioprofessionnelle, article 5, Namur, 18/07/2002 : commentaires des articles, p. $3 / 7$. le repérage des compétences et leviers d'action, l'analyse des motivations professionnelles, la (co)construction de projet(s), la mise en œuvre de stratégies d'approche du marché, la clôture du dossier.

(1) L'accueil par le CAP s'opère généralement dans la foulée de l'inscription du demandeur d'emploi par un conseiller en démarches administratives (CDA), au terme d'une séance d'information collective sur les services aux particuliers du FOREM. Autrement dit, les CAP ne sont pas les spécialistes de l'accueil ; cette spécialité incombe au CDA, ce spécialiste des droits sociaux et des procédures administratives. Cette phase de prise de contact permet au CAP de présenter sa fonction (personne de référence tout au long du parcours), le dispositif réglementaire dans lequel s'inscrit le demandeur (Plan Jeunes +, Programme de Transition Professionnelle, suivi volontaire, etc.) et les modalités de mise en œuvre de ce dispositif (contractualisation, suivi, rapport final, procédures d'objectivation, sanctions éventuelles).

(2) À la suite de cette phase d'accueil, le CAP s'efforce de repérer les éventuels besoins du demandeur d'emploi dans le cadre de son insertion socioprofessionnelle. Le CAP dispose d'une «grille de pré-repérage » qui constituera un tableau de bord de l'évolution de son « patient ». Cette grille est utilisée comme un cadre très général dans lequel se déroule l'entretien; elle permet d'organiser l'information recueillie auprès du demandeur. L'agent dose, le plus habilement possible, rapports d'intéressement et rapports d'évaluation ${ }^{12}$ : d'une part, l'agent procède au contrôle d'un certain nombre de paramètres et d'actions (inscription, CV, société d'intérim, permis de conduire...), tâche indispensable pour caractériser la situation du demandeur d'emploi, pour poser un diagnostic ; d'autre part, il essaie d'amorcer une relation de confiance, nécessaire pour tenter d'inciter le demandeur à s'impliquer dans un travail sur sa propre employabilité.

\footnotetext{
${ }^{12}$ Ces deux formes d'ajustement ont été mises en évidence par Eymard-Duvernay et Marchal (1994). Ces auteurs définissent les dispositifs d'intéressement comme la prise en compte de l'intérêt des personnes que l'on cherche à mobiliser dans le cadre d'une négociation, et les dispositifs d'évaluation comme l'évaluation de la conformité des conduites en référence à des principes généraux (Eymard-Duvernay et Marchal, 1994, p. 15).
} 
(3) Le CAP s'attache à définir les étapes à franchir par le demandeur d'emploi, au long de son «trajet d'employabilité », en fonction de sa demande initiale $^{13}$ et des enseignements du pré-repérage. Le CAP approfondit l'analyse des besoins du demandeur d'emploi, convient avec lui d'une hiérarchisation des besoins et définit un plan d'action. Il est clairement attendu du CAP qu'il «motive à l'action », qu'il implique fortement le demandeur dans le travail qu'il opère sur lui-même, sur sa propre employabilité.

(4) Il est également attendu du CAP qu'il repère l'ensemble des compétences professionnelles et extra-professionnelles du demandeur d'emploi en termes de savoir, savoir-faire et savoir-être. À ce stade, le repérage des compétences s'opère en dehors de toute considération de projet(s) professionnel(s). Le CAP complète son analyse par l'utilisation du ROME de l'ANPE (Répertoire opérationnel des métiers et des emplois : fiches métiers et aires de mobilité professionnelle). Le CAP procède aussi au repérage de certaines aptitudes supplémentaires pour un projet d'employabilité. Rappelons que le CAP n'est pas spécialiste du repérage des compétences; le spécialiste étant le conseiller en orientation professionnelle (COP). Toutefois, en bon généraliste, le CAP doit amorcer cette phase de repérage et la mener jusqu'aux limites de son propre métier de généraliste. En clair, toute situation critique ou blocage nécessitera l'intervention d'un COP ; cette intervention ponctuelle viendra se greffer sur l'action générale du CAP.

(5) Le CAP dispose également d'une grille d'analyse des motivations. L'analyse des motivations du demandeur d'emploi doit permettre d'établir des convergences ou divergences entre projet de vie, projet professionnel et réalité du marché.

(6) Dans la phase de (co)-construction de projet(s), le CAP fait choisir à l'usager trois projets professionnels à partir d'une liste de familles professionnelles ; il édite les fiches ROME correspondantes et compare

\footnotetext{
${ }^{13}$ Rappelons que cette démarche est obligatoire pour tout demandeur d'emploi inscrit à l'office régional (FOREM); un refus de participation à ces mesures "actives" d'accompagnement est automatiquement sanctionné par l'ONEM (la sanction pouvant aller jusqu'à la perte des droits aux allocations de chômage).
}

les contenus de ces fiches avec les grilles d'analyse des compétences et leviers d'actions ainsi que la grille d'analyse des motivations ( $c f$. supra). Une phase de mise en œuvre de stratégies d'approche du marché est initiée par le CAP mais, à nouveau, celuici n'étant pas spécialiste en la matière, il aiguillera souvent le demandeur d'emploi vers les conseillers en recherche d'emploi (CRE), ces spécialistes des techniques et stratégies d'approche du marché de l'emploi.

(7) Tout au long de l'accompagnement du demandeur d'emploi, ce « trajet d'employabilité », le CAP garde des traces du suivi sur différents supports ${ }^{14}$. L'accompagnement du demandeur d'emploi se clôture par un rapport (positif ou négatif) transmis à l'Office national de l'emploi (ONEM) chargé du contrôle et de l'indemnisation des chômeurs au niveau de l'ensemble de la Belgique (voir note de bas de page $n^{\circ} 7$ ).

\section{LES CONSEILLERS EN FORMATION : SPÉCIALISTES DE L'EMPLOYABILITÉ}

Le dispositif « Carrefour Formation » date de la mise en place du Parcours d'insertion en Région wallonne ${ }^{15}$. La Convention cadre définit deux missions spécifiques qui incombent aux professionnels des Carrefours Formation. D'une part, une mission d'information : œuvrer à la transparence de

\footnotetext{
${ }^{14}$ D'une part, il constitue un dossier (support papier) contenant : le formulaire A36 d'inscription complété par le CDA, la convention d'insertion qui contractualise l'accompagnement (formulaire $\mathrm{CAPJ}+$ ), la grille de pré-repérage en 4 axes, une note mémo (portant la mention: " notre expertise ne consiste pas à trouver une solution rapide ! »), des notes prises par l'agent lors des entretiens de suivi, d'éventuels $\mathrm{CV}$ du demandeur d'emploi réalisés aux Tables de l'emploi (service du FOREM spécialisé dans les techniques d'écriture et de constitution de dossiers de candidature). D'autre part, le CAP encode chacune de ses interventions dans la base de données ERASME (support informatique).

${ }^{15} \mathrm{La}$ Convention cadre des Carrefours Formation et son annexe "Charte de l'animation pédagogique " sont signées le 15 janvier 1998 par le ministre du Budget, des Finances, de l'Emploi et de la Formation et les représentants des différents opérateurs partenaires, à savoir l'Agence wallonne pour l'intégration des personnes handicapées (AWIPH), les Organismes d'insertion socioprofessionnelle/ Entreprises de Formation par le Travail (OISP/EFT), l'Enseignement de promotion sociale (ESP), l'Institut de formation des petites et moyennes entreprises (IFPME) et le FOREM.
} 
l'offre et à la diffusion de l'information sur les actions de formation / insertion, auprès des personnes et des opérateurs de terrain. D'autre part, une mission de conseil : écouter, comprendre et enregistrer la demande du candidat, et l'aider à définir son plan d'action en tenant compte de sa situation et des possibilités de formations existantes.

Le Gouvernement wallon accorde une licence (Hughes, op. cit.) aux professionnels de Carrefour Formation - mais, ici, de manière non exclusive -, prévoyant la coexistence de plusieurs organisations et professionnels sur un même (sous-)secteur, celui de l'accueil et de l'information des bénéficiaires du dispositif intégré d'insertion. Les conseillers de Carrefour Formation partagent donc ce territoire avec d'autres groupes professionnels, ceux des Maisons de l'Emploi et des Espaces Ressources Emploi du FOREM et des services d'accueil et d'information d'autres organisations partenaires.

Le conseiller en formation de Carrefour Formation se présente comme un des spécialistes de l'employabilité : il se définit comme le spécialiste de l'information sur les formations :

«Je dirais que la fonction de CAP, c'est un peu le généraliste et la fonction de conseiller Carrefour, ça serait un peu le spécialiste, dans le sens où le généraliste fait un diagnostic et parfois est amené à envoyer chez le spécialiste pour compléter son diagnostic. (...) Quand les gens viennent à Carrefour, ils veulent surtout faire appel à notre expertise au niveau de la formation ». (Entretien agent $\mathrm{CF}$ FOREM, Liège)

Toutefois, ce profil d'expert en information sur les formations est loin de résumer l'ensemble des compétences de ce professionnel, eu égard à la diversité des publics avec lesquels il travaille, au caractère fluide et non stabilisé de l'information qu'il traite, au cadre organisationnel très particulier dans lequel il évolue. En effet, Carrefour Formation multi-opérateur se présente comme une organisation hybride à l'extrême, au carrefour de cinq opérateurs de formation, qui «tient ensemble » d'une part, grâce à un travail de décloisonnement organisationnel fort, opéré par les acteurs de terrain, et d'autre part, au moyen de procédés organisationnels $d^{\prime}$ ' indivision sociale du travail ». Dès lors, le métier qu'exercent ces professionnels se situe également au croisement de différents métiers: l'expert en formation, le psychologue, le documentaliste et l'agent de réseau.

Pour comprendre le travail des conseillers à Carrefour Formation, il est indispensable de s'intéresser de près à la configuration des lieux, aux territoires : porter une attention extrême aux mouvements d'entrée et de sortie, aux types d'énoncés, aux codes, aux postures affectés à chaque espace... Contrairement à ce que nous avons pu observer dans le service des Conseillers en Accompagnement Professionnel du FOREM (Orianne, 2005a), où le travail et les modes opératoires sont principalement définis par des règles (lois, arrêtés, décrets, règlements administratifs, ...), à Carrefour Formation, les cadres de travail ne sont pas des cadres réglementaires mais des cadres "géographiques» (localisation physique des activités): c'est principalement l'espace qui définit le travail et les modes opératoires (Orianne, 2006b). La structure partenariale de cette organisation explique le rapport instrumental à la règle administrative et à l'autorité : la prescription du travail, les directives sont négociées entre les partenaires, chaque agent dépend de deux voies hiérarchiques ${ }^{16}$; le gestionnaire n'a pas de pouvoir hiérarchique sur les membres de l'équipe... À Carrefour Formation, tout espace de travail est un espace construit en commun, une «zone neutre» entre différents opérateurs de formation concurrents.

Le travail de l'agent avec le public s'opère dans trois cadres d'interaction très différents : l'accueil en salle de documentation (travail en flux continu dans l'espace ouvert), l'entretien conseil (dans un bureau, relation de face-à-face, sur rendez-vous), les séances d'information collective (animations de groupes programmées, gestion des dynamiques collectives).

Le premier accueil dans l'espace ouvert s'effectue généralement debout. L'agent se présente, présente brièvement Carrefour Formation: le service de documentation (l'offre de formation classée en 20 domaines, les ordinateurs et photocopieuses à

\footnotetext{
${ }^{16}$ D'une part, la hiérarchie Carrefour : le Comité d'accompagnement des Carrefours Formation (CACF) ; d'autre part, la hiérarchie de l'opérateur partenaire : un des cinq opérateurs partenaires du dispositif Carrefour Formation (FOREM, AWIPH, etc.).
} 
disposition du public), le service d'entretien conseil et les séances d'information collective programmées. Souvent, l'agent sera amené à préciser que «Carrefour Formation, ce n'est pas le FOREM, ni l'ONEM», qu'il ne s'agit pas non plus d'un centre de formation mais d'un centre de documentation et de conseil sur les formations. Ces premières balises étant posées, l'agent sort les dossiers correspondant à la demande de l'usager et s'assied avec lui à une table. L'agent essaye de décoder la demande de l'usager ou, le cas échéant, de faire émerger cette demande, ou de la retraduire. L'agent parcourt les dossiers et, en fonction du degré d'autonomie de l'usager, en explique le contenu: la table des matières, les informations sur les métiers, les informations sur les formations. Ensuite, l'agent procède à une présentation - la plus complète, la plus neutre, et la plus adaptée possible - des différentes filières de formations existantes: les différents opérateurs, les modalités d'accès, les diplômes ou certifications délivrés, les contenus, les types de pédagogie... Enfin, l'agent sélectionnera avec l'usager les " produits » qui conviennent le mieux à ses besoins et à sa situation ; il proposera généralement de photocopier les informations retenues et suggérera à l'usager de prendre une série d'informations complémentaires par téléphone ou via Internet. À ce stade, l'usager peut, s'il le souhaite ou s'il en ressent le besoin, prendre rendez-vous pour bénéficier d'un entretien-conseil individualisé.

Dans le cadre plus individualisé de l'entretien conseil, l'agent procédera à une analyse plus approfondie de la demande. Cette analyse consiste théoriquement, si l'on s'en tient au Vade Mecum de l'analyse de la demande ${ }^{17}$, en une analyse de faisabilité du projet (pour autant que l'usager arrive avec un projet professionnel relativement défini) : 1) évaluer l'adéquation entre le projet du candidat et ses compétences, ses aptitudes physiques, sa personnalité, son cadre de vie ; 2) évaluer l'adéquation entre le projet et l'emploi visé (qu'il soit disponible ou non);

\footnotetext{
${ }^{17}$ Ce Vade Mecum est un outil de travail importé du FOREM (des Centres d'orientation et d'insertion du FOREM : les COISP) et n'a jamais été remis officiellement aux conseillers de Carrefour Formation. Cependant, par "initiative personnelle», certains anciens membres des COISP, travaillant depuis à Carrefour, ont introduit ce document au sein des équipes pour outiller leurs nouveaux collègues, souvent démunis face à l'entretien conseil.
}

3) évaluer l'adaptation du candidat aux conditions de travail (de cet emploi « théorique »); 4) confronter le projet professionnel au projet de formation. Lorsque le projet n'est pas défini, le travail d'analyse de la demande sera essentiellement exploratoire et consistera à faire émerger des projets, à traduire des envies, des craintes, des silences... en projets. Dans ces situations critiques où les outils de travail deviennent inopérants, les professionnels se fient à leur expérience et à leurs intuitions ; ils s'appuient également sur le principe éthique de responsabilisation de l'usager, d'activation de ses facultés d'autonomie : c'est à lui de faire son chemin, c'est à lui de trouver la solution.

Les conseillers de Carrefour Formation organisent également des séances d'information collective, soit dans leur propre bâtiment, soit à l'extérieur chez des partenaires (opérateurs de formation, centres publics d'action sociale...). On peut distinguer au moins deux types de séances d'information collective: celles qui visent la présentation de familles professionnelles et/ou de filières de formation et celles qui concernent la présentation de Carrefour Formation.

\section{LES FORMATEURS DES ENTREPRISES DE FORMATION PAR LE TRAVAIL : GÉNÉRALISTES POUR PUBLICS SPÉCIALISÉS}

Constituées sous forme d'Association sans but lucratif (ASBL, $c f$. loi du 27 juin 1921) ou développées par des Centres publics d'action sociale ${ }^{18}$ (CPAS, $c f$. loi organique du 8 juillet 1976), les Entreprises de Formation par le Travail (EFT) proposent des formations, d'une durée maximale de dix-huit mois, pour des stagiaires faiblement qualifiés et en grandes difficultés ${ }^{19}$. Ces actions de formation se

\footnotetext{
${ }^{18}$ Leur mission est d'assurer aux personnes et aux familles en difficulté un revenu minimum d'existence (le minimex, qui s'apparente au RMI - revenu minimum d'insertion - en France).

${ }^{19}$ Le secteur des EFT/OISP se compose de cinq fédérations qui réunissent les différents centres tout en respectant les options philosophiques, pédagogiques et économiques de chacun; une interfédération rassemble les cinq fédérations et assure un rôle d'interlocuteur unique auprès des pouvoirs publics.
} 
caractérisent par une pédagogie spécifique alliant formation théorique, travail productif et accompagnement psychosocial (Fusulier, 1996).

Le Gouvernement wallon accorde aux travailleurs en EFT une licence exclusive pour exercer leur activité professionnelle ${ }^{20}$. Il définit différents objectifs généraux (préparer l'insertion socioprofessionnelle, favoriser l'égalité des chances, ...) et d'autres objectifs intermédiaires (autonomie sociale, citoyenneté, projets professionnels, accès à la formation qualifiante, ...). En procédant de la sorte, le Gouvernement wallon affirme implicitement le caractère linéaire du dispositif intégré d'insertion, en reléguant le secteur associatif des EFT/OISP dans une phase de préqualification, de préparation des stagiaires à la formation qualifiante : en clair, la formation par le travail ne devrait déboucher ni sur l'emploi (un poste de travail) ni sur une certification (un diplôme, un titre); elle doit préparer les stagiaires à l'entrée dans les centres de formation du FOREM ou dans l'enseignement de promotion sociale.

Le professionnel d'une EFT exerce un métier de " généraliste» pour un public «spécialisé»: un métier de généraliste dans le sens où il dispense une formation très générale, pour public spécialisé dans la mesure où même le Gouvernement wallon a bien $\mathrm{du}$ mal à définir ce public «dont personne ne veut... », ces individus qui présenteraient les plus sérieux troubles de l'employabilité :

«On doit être des généralistes. Puisque dans le cadre de la formation, on touche à tout, et donc il faut avoir un très, très bon réseau. Le stagiaire n'est ici que pour 18 mois (...) Je vais essayer de faire une accroche avec le service de médiation de dettes, puis faire une accroche avec le service de droit au logement, puis avec un service de santé mentale par exemple, ainsi de suite,... On a beaucoup de stagiaires qui sont isolés ou atomisés. (...) Je pense qu'on est le seul contact humain qu'ils aient, on est les premiers à se rendre compte s'ils viennent à mourir du jour au lendemain. Donc, on va essayer de renouer des contacts et de les relancer dans une autre dynamique ». (Entretien Directeur EFT, Liège)

${ }^{20}$ Gouvernement wallon, décret du 01/04/2004 relatif à l'agrément et au subventionnement des EFT/OISP, Namur.
Le travail en EFT se caractérise par une telle omniprésence du stagiaire au sein de l'organisation que c'est lui qui redéfinit constamment l'organisation, qui la construit et construit les modes opératoires du travail d'insertion ${ }^{21}$. De manière infiniment moins formalisée que pour les CAP du FOREM, le travail d'insertion en EFT se compose globalement de quatre phases distinctes dont l'ampleur varie fortement d'un stagiaire à l'autre, d'une situation à l'autre. La linéarité de cet agencement en quatre phases n'a pas été démontrée : de nombreux parcours individuels de stagiaires se caractérisent par des « allers-retours » entre ces différentes phases.

Le travail d'insertion commence par une phase d' "apprivoisement» mutuel entre le stagiaire et le professionnel, centrée sur un processus de stabilisation des «chutes» (ou décrochages) dans des « zones d'expérimentation et de respect mutuel »,pour reprendre le terme indigène. La stabilisation des décrochages nécessite la réévaluation permanente des addictions, dettes, absence de domicile fixe, troubles du comportement, etc. Dans un deuxième temps, le professionnel développe des rapports d'intéressement afin d'impliquer progressivement le stagiaire dans la construction de son parcours (pratiques de mise en situation professionnelle et d'auto-évaluation des comportements et des réalisations des stagiaires). La phase d'autonomie constitue la troisième phase du travail d'insertion en EFT. Enfin, la dernière étape correspond au travail sur l'employabilité. C'est à ce stade ultime que tout ce qui a été opéré en amont par le professionnel de l'insertion peut déboucher sur le travail proprement dit de l'employabilité du stagiaire. Tout ce qui a été enseigné comme comportement social de base va servir au stagiaire dans ses comportements professionnels ; fort de l'expérience relationnelle acquise (espaces de respect mutuel), le stagiaire pourra progresser dans le champ professionnel (sur un chantier, en entreprise, seul dans un véhicule...);

\footnotetext{
${ }^{21}$ Notons à ce propos que, dans chacune des EFT, les stagiaires de la filière bâtiment contribuent directement à la construction des locaux. Plus globalement, les stagiaires participent à la production de richesses, même non-rentable, qui permet l'octroi de subsides. Ils participent, en outre, à la construction des règles et outils de travail. D'autres éléments indiquent, enfin, que les stagiaires participent à la construction des programmes de formation et des outils d'évaluation pédagogique (par la pratique assidue de l'autoévaluation du stagiaire).
} 
il pourra appliquer les techniques de recherche d'emploi sur la base du travail de recherche active de soi, également opéré en amont.

\section{L'ORIENTATION CLINIQUE DU TRAVAIL D'INSERTION}

Les trois groupes professionnels étudiés visent moins directement la mise à l'emploi qu'un travail sur l'employabilité du demandeur d'emploi : leur travail consiste à motiver, intéresser l'individu à se mettre au travail sur lui-même. Rappelons à cet égard que l'employabilité constitue un des objectifs officiels des politiques actives (européennes, fédérales, régionales) de l'emploi. Notons également que cette finalité semble la seule viable sur un plan moral pour ces agents de première ligne. En effet, n'ayant de pouvoir d'action ni au niveau de la création d'emploi, ni au niveau de l'accès à l'emploi, c'est sur un autre plan que doit se situer leur intervention. N'étant pas responsables de l'emploi ou du nonemploi des individus, ils responsabilisent ces derniers à travailler leur employabilité, leur capacité à trouver un emploi, dans le cadre d'entretiens individualisés ou de séances d'information collectives, de groupes de discussion ou d'ateliers d'écriture de lettres de motivation et de $\mathrm{CV}$, de simulations d'entretiens d'embauche filmées, de mises en situation professionnelle, etc. L'autonomie de la personne en recherche d'emploi apparaît comme l'objectif principal du travail d'insertion, comme en témoignent ces extraits d'entretien :

\section{FOREM :}

«C'est l'autonomie dans la recherche d'emploi qui est visée » (CAP, Liège)

"L'autonomie de la personne en recherche d'emploi : c'est une finalité. (...) Tu sais... c'est redonner confiance, leur proposer de devenir acteurs... Et certains le sont déjà...» (Responsable CAP, Libramont)

"C'est essayer de les rendre autonomes tout simplement. De leur dire: mais voilà, maintenant tu as toutes les cartes en main... voilà, à toi de jouer.» (CAP, Libramont)

\section{Carrefour Formation :}

«Nos actions sont conçues dans l'optique du développement et de l'encouragement à l'autonomie, dans l'espace ouvert, comme dans les demandes à réaliser par les usagers" (Rapport d'évaluation semestriel, juillet-décembre 1998, Liège, p. 10).

« Je crois au fond ici... je sais que nous ici aussi on a un objectif d'autonomie, on aimerait que les gens soient autonomes. Mais il ne faut pas confondre autonomie avec abandon et désintérêt pour la personne qui est là. Sous prétexte qu'il doit être autonome, tu ne le laisses pas là en plan devant les fardes $^{22}$. 》 (Entretien agent CF IFPME, Liège)

"C'est bien de vouloir des personnes autonomes, mais il faut leur donner les moyens d'être autonomes. On ne les rend pas autonomes comme ça en claquant des doigts. » (Entretien agent CF FOREM, Liège)

«Il y a un apprentissage, moi je crois. On ne te fait pas une intraveineuse d'autonomie.» (Entretien agent CF EPS, Liège)

\section{Entreprise de Formation par le Travail :}

«L'objectif général poursuivi réside bien entendu dans l'acquisition d'une autonomie personnelle, d'une socialisation au monde du travail. (...) Cela rend une approche individuelle nécessaire: "faire $d u$ sur mesure". » (Le Trusquin EFT, rapport d'activité 1998, p. 6)

«L'objectif, c'est de permettre à des jeunes de trouver ce qu'ils veulent faire, d'être autonomes dans leur recherche d'emploi.» (Entretien Formatrice EFT, Marloie)

Ce travail sur l'employabilité de la personne, en vue de renforcer son autonomie dans sa recherche d'emploi, s'opère à distance de la sanction administrative de l'État (qui reste une compétence fédérale). Le sens du travail d'accompagnement, en Région wallonne, se construit en rupture avec toute conception coercitive du métier, malgré le caractère contraignant du dispositif: d'une part, les agents se distinguent avec insistance des «facilitateurs》 de l'ONEM, ces agents chargés du contrôle des

22 Il s'agit des classeurs contenant la documentation sur les formations. 
bénéficiaires de l'assurance-chômage ; d'autre part, ils s'opposent avec force à toute forme d'évaluation externe de leur travail de diagnostic et de traitement (en termes de ratios, de taux d'insertion ou de mesures d'objectivation): "nous, on ne fait pas $d u$ quantitatif, (...) on privilégie la qualité sur la quantité », ne cessent-ils de répéter.

Pour amener le demandeur d'emploi à opérer ce travail sur lui-même, les agents dosent habilement des rapports d'intéressement et des rapports d'évaluation (Eymard-Duvernay, Marchal, op. cit.) : cette double composante du travail d'insertion (l'aide et le contrôle) est liée à la nature du mandat politique (l'activation d'individus). Mais par quel procédé parviennent-ils effectivement, dans le cadre de dispositifs contraignants d'accompagnement de demandeurs d'emploi, à intéresser l'individu qui cherche un emploi à se mettre au travail sur luimême? Selon nous, cet appariement - entre une demande d'emploi et une offre de mise au travail sur soi - repose sur un «savoir ésotérique» (non profane) que détient et développe la profession, une capacité collective (technique et morale) à transformer, traduire de la souffrance en «troubles » de l'employabilité. Ces pratiques professionnelles s'alimentent d'une croyance commune en l'existence de troubles individuels de l'employabilité, indépendante de leur construction sociale (Orianne, 2006a), - comme si ces troubles existaient dans la nature des choses et des êtres.

Les travaux de Freidson sur la profession médicale (1984) nous invitent à envisager ces diagnostics apparemment neutres (caractéristique principale d'une profession consultante), qu'opèrent généralistes et spécialistes de l'employabilité, comme un travail "positif» de construction de troubles de l'employabilité (repérage et identification de symptômes, production de diagnostics, naturalisation des catégories). Accomplissement pratique des politiques actives d'emploi, cette nouvelle profession consultante dans le champ de l'insertion oriente le traitement des chômeurs vers une voie résolument clinique : cette profession construit (catégorise) des troubles de l'employabilité ; elle organise l'expérience de ces troubles et la socialisation au rôle de «malade» (comment se comporter comme chômeur actif ?) ; ainsi, elle fonde sa légitimité profession- nelle tout en rendant possible la responsabilisation du chômeur (Orianne, 2005b).

Le diagnostic et le traitement apparaissent comme les deux actes fondamentaux de la pratique clinique des travailleurs de l'insertion. Comme l'avance Baszanger (1986) pour les médecins, le diagnostic est central dans leur travail; en effet, il "permet l'organisation des interventions à venir. Il est aussi central dans ses conséquences sociales puisqu'il introduit une nouvelle situation: celle de malade, reconnue sur des bases communément admises (le savoir médical) » (Baszanger, 1986, p. 13). De plus, le diagnostic apparaît invariablement comme un "consensus normatif», un arrangement toujours singulier, auquel parviennent spécialistes et profanes (usagers), médecin et malade. Il s'agit d'un processus de négociation sous contraintes normatives, où l'usager (le chômeur, le malade...) fait partie intégrante de la division du travail et contribue en particulier au travail de catégorisation. En effet, les troubles de l'employabilité ne constituent pas des données intangibles, ils ne se construisent pas une fois pour toutes : ils sont négociés et résultent d'une succession d'interactions dans le temps.

C'est le cas de Martine (27 ans) qui, au terme de cinq mois de formation en EFT, est diagnostiquée « immature» et « irresponsable»: ce verdict sans appel, qui met un terme à sa formation, est le résultat d'une longue et douloureuse négociation entre Martine, les formateurs de l'EFT et son maître de stage, - comme en témoignent les différentes pièces qui composent le « dossier stagiaire » de Martine. Le rapport d'évaluation finale stipule :

«Martine ne sait toujours pas ce qu'elle veut exercer comme métier. Tout ce qu'elle sait, c'est que ce ne sera pas comme chauffeur-livreur. Pour le reste, nous pensons que Martine peut suivre toutes les formations qu'elle veut, c'est inutile tant qu'elle ne choisit pas une orientation professionnelle. De plus, des compétences, elle en a. Ce qui lui manque, c'est une attitude adulte et responsable. Cela ne s'apprend pas avec des formations. » (Rapport d'évaluation finale de Martine, EFT Marloie)

C'est aussi le cas de Jean-Pascal (22 ans). Après plusieurs tentatives, il ne parvient toujours pas à 
écrire son $\mathrm{CV}$ selon les attentes peu explicites de son CAP : il se voit, dès lors, orienter vers les Tables de l'emploi, ce service du FOREM spécialisé dans les techniques d'écriture et de constitution de dossiers de candidature. La simulation d'entretiens d'embauche filmée (et commentée) lui a également été conseillée, dans un second temps, pour pallier cette " inexpérience », qu'il reconnaît bien volontiers.

C'est encore le cas de Mireille (la quarantaine) qui ne parvient pas à «faire son deuil» (dit-elle) ou à «décrocher» (dit le conseiller) du travail intérimaire, ce travail sans qualité dont elle se contente depuis trop longtemps, et qui l'empêche de définir un projet professionnel «cohérent» et «épanouissant» (selon les termes du conseiller de Carrefour Formation). Ce dernier, après deux heures d'entretien, l'invite donc à renoncer au travail précaire en travaillant sur elle-même, en prenant le «temps de se connaître » (dit-il), dans le cadre, par exemple, des activités que proposent les conseillers en orientation professionnelle du FOREM.

Quant à Jean-Pierre (32 ans), rapidement étiqueté « incapable de se lever le matin» (et plus globalement «de respecter les horaires») par ses formateurs lors des premières évaluations, il travaille principalement le sens du rythme et l'autodiscipline dans le cadre de sa formation aux métiers du bâtiment au sein d'une EFT à Liège.

À l'image des maladies chroniques, les troubles de l'employabilité apparaissent alors comme une pathologie dominante (de masse) et incertaine (cause, durée, issue), qu'il convient de gérer, plus que de guérir. L'incertitude des catégories cliniques utilisées par les travailleurs de l'insertion renforce le poids des négociations incessantes ${ }^{23}$. Cependant, bien que le diagnostic résulte d'une négociation, il convient de

\footnotetext{
${ }^{23}$ Notons que les professionnels de l'insertion ne s'appuient sur aucune étude nosographique et ne contribuent en aucune manière à la production de telles études. Il ne s'agit pas d'une profession savante, mais bien d'une profession consultante, en devenir, qui pratique le diagnostic en l'absence d'une nosologie des troubles de l'employabilité, qui repère et catégorise des symptômes en l'absence de catégorisation établie. Cependant, dans le cadre de réunions de travail, en «coulisses » ou derrière la scène des interactions de face-à-face avec le public, ces professionnels œuvrent à la construction d'un savoir pratique, œuvrent à la stabilisation de catégories de « troubles » qui émergent de la pratique d'accompagnement de chômeurs (par exemple, les troubles du comportement, de l'orientation, de la motivation, du langage, etc.).
}

rappeler que les deux intervenants n'ont pas le même poids dans la décision finale : le «spécialiste»se différencie du profane, sur la base de son expertise, de son expérience en matière de diagnostic, de son jugement sur les moyens d'établir le diagnostic (Strauss, 1992, p. 163).

Les marges de manœuvre de l'usager, sa place dans la division du travail clinique, varient fortement d'un territoire professionnel à l'autre: au FOREM, les marges sont relativement étroites et principalement balisées par des règles administratives (énoncés des politiques et traductions organisationnelles : le vademecum). En EFT, la place de l'usager nous apparaît au centre de la division sociale du travail d'insertion ou d'activation.

Cependant, indépendamment du cadre organisationnel, les marges de manœuvre de l'usager dépendent de compétences communicationnelles. L'usager doit impérativement en faire preuve, ou doit les acquérir rapidement, pour prendre place effectivement dans la division sociale du travail de catégorisation. La production négociée de catégories cliniques est avant tout une activité langagière qui implique, de la part des acteurs, la maîtrise de codes, d'énoncés, de styles d'énoncés (permettant de se présenter dans une relation de face-à-face, au téléphone ou par écrit, contribuant à se mettre en valeur, émouvoir, convaincre, etc.). Plus que le projet professionnel de l'usager, c'est la manière dont il en parle qui importe, qui compte pour le professionnel de l'employabilité. Plus que les compétences techniques, que ces agents ne sont souvent pas en mesure d'apprécier à leur juste valeur, c'est bien leur mise en mots qui oriente la mise en œuvre de l'action.

En définitive, dès que le chômeur pénètre l'univers professionnel des travailleurs de l'insertion, la profession impose une organisation de l'expérience de troubles de l'employabilité ainsi qu'une socialisation au rôle de «demandeur d'emploi actif et autonome» (Orianne, op. cit.). Le travailleur de l'insertion, nouvelle forme d'entrepreneur moral (Freidson), accomplit des fonctions de contrôle social (régulation et socialisation) en œuvrant à l'intériorisation de normes à grande échelle. Sa tâche quotidienne est d'affecter une étiquette " clinique » à des symptômes que des profanes ont déjà repérés 
comme indésirables, transformer en un problème grave ce qu'un profane voyait comme un problème mineur, transformer des symptômes en maladie (en tant que rôle social). La progression de l'usager à travers les différentes catégories de déviance ou de troubles de l'employabilité peut alors être envisagée comme une carrière (Goffman, 1968, pp. 177-225 ; Freidson, 1984, p. 246) : un travail sur soi à temps plein et à durée indéterminée ${ }^{24}$.

Précisons enfin que le traitement clinique du chômage n'est pas synonyme d'une logique occupationnelle ou d'un traitement social de certaines catégories de chômeurs «inemployables» (Dugué et Verger, 2003) ou "insérés permanents» (Castel, 1995). Le traitement clinique est transversal à tous les dispositifs d'insertion et s'adresse à toute personne sans emploi, à tout actif inoccupé. S'il a des effets catégorisants sur les demandeurs d'emploi, il les inscrit simultanément dans une logique d'inclusion sociale extrêmement poussée, qui tend à « socialiser» au marché du travail l'ensemble de la population active, jusque dans ses moindres recoins. La construction (ou catégorisation) de troubles de l'employabilité n'est donc pas nécessairement ou seulement une mise en inaptitude de certaines catégories de chômeurs, un nouvel outil de gestion des files d'attentes sur le marché du travail (Le Bianic, in Dugué et Verger, 2003) ; elle est une mise en projet de l'ensemble de la population active, un projet de « société du travail sur soi » (Vrancken et Macquet, 2006).

\section{TROIS FACCONS DE « CONSTRUIRE » DES TROUBLES DE L'EMPLOYABILITÉ}

Par-delà le consensus normatif sur l'orientation clinique du travail d'insertion, les pratiques des intermédiaires publics du marché du travail varient sensiblement en fonction des cadres organisationnels (au sens large) dans lesquels ils opèrent : soit une institution telle que le Service public d'emploi (FOREM), soit une organisation en réseau telle que Carrefour

${ }^{24}$ Par l'incertitude qui pèse sur l'insertion réelle des personnes sans emploi en Région wallonne.
Formation, soit une Entreprise de Formation par le Travail qui valorise les cadres de l'interaction, de la rencontre intersubjective. Nous empruntons à la littérature « conventionnaliste », consacrée aux intermédiaires du marché du travail, une typologie des régimes d'actions pour situer les différentes modalités de construction d'un trouble de l'employabilité : le cadre de l'institution (pour le conseiller du FOREM), du réseau (pour le conseiller de Carrefour Formation), de l'interaction (pour le formateur en EFT) et, enfin, celui du marché (comme modèle repoussoir commun).

Eymard-Duvernay et Marchal (1997) proposent une cartographie des régimes d'action des intermédiaires du marché du travail, permettant de différencier quatre façons de recruter, quatre conventions de compétence ou cadres entre lesquels le « recruteur» doit arbitrer, dès lors qu'il s'agit d'établir une compétence. Dans le cadre d'une institution, le jugement est référé à des règles (toujours incomplètes, nécessitant donc un travail d'interprétation) qui mettent en relation des catégories générales et des objets ; le recruteur apparaît comme un régulateur et le candidat est « traduit» par sa qualification. Dans la convention de marché, le recruteur est un sélectionneur qui intensifie la concurrence entre les candidats et tente d'évaluer, et donc de ne retenir que les aptitudes individuelles. Dans la convention de réseau, le recruteur apparaît comme un médiateur qui considère que les compétences des acteurs sont distribuées dans un réseau de relations. Enfin, dans le cadre de l'interaction, dans une relation de face-àface, le recruteur cherche à établir une relation de confiance en prenant appui sur son intuition, et le jugement apparaît comme un ordre négocié - au sens de Strauss (1992) - un arrangement local, produit émergeant de la configuration relationnelle de face-àface. Dans ce cadre, les compétences sont émergentes car elles résultent du déroulement de l'interaction. Cette typologie nous permet de situer l'action spécifique de chacun des trois groupes professionnels étudiés en Région wallonne.

Le conseiller en accompagnement professionnel s'inscrit pleinement dans le cadre de l'institution. L'évaluation de l'employabilité s'effectue principalement au travers de dispositifs de mise en équivalence : faire entrer les «clients» du FOREM 
dans les catégories générales et abstraites des politiques actives d'emploi. La construction de « troubles de l'employabilité » s'opère au départ d'un travail de comparaison et de mise en équivalence à partir de divers référentiels (référentiel Rome, grille de compétences, etc.) servant de supports au jugement, au diagnostic. Quant aux conseils prodigués, ils consistent à intéresser suffisamment le demandeur d'emploi pour qu'il accepte d'entrer dans ce jeu de catégorisation, qui lui assignera une place potentielle dans le cadre des référentiels métiers ou des référentiels de compétences. La construction de la norme d'employabilité s'appuie ici largement sur divers outils standards de catégorisation des emplois, qualifications et compétences; il s'agit d'une norme réglementaire et standardisée qui sous-tend les jugements et choix singuliers proposés à chaque «client»du SPE. Les agents du FOREM mettent donc essentiellement en œuvre des cadres conventionnels circonscrits par des textes réglementaires, des répertoires de métiers ou de formations, dont ils modulent l'application à la situation de la personne. La capacité à poser un jugement et à faire un usage circonstancié de ces cadres réglementaires et institutionnels est ainsi décisive chez le professionnel. De plus, cette capacité doit se conjuguer avec une compétence à construire une interaction et une empathie réussie avec son client, relation de face-à-face, cruciale pour susciter l'intéressement de la personne, mais aussi pour disposer de suffisamment d'informations sur chaque cas singulier. Cependant, l'interaction est courte et largement cadrée par les procédures, les outils, les espaces de travail dans lesquels elle se déploie.

À l'opposé, des interactions longues s'observent entre les travailleurs sociaux des EFT et le stagiaire, par son insertion de facto dans un collectif de travail, par sa mobilisation dans un parcours de mises en situation sociale et technique, dont l'enjeu est autant pédagogique et thérapeutique que productif. De ce fait, la norme d'employabilité s'éprouve par le stagiaire dans le concret des mises en jeu de soi dans le travail technique et la coopération sociale. L'interaction de longue durée, le soutien psycho-social, l'encadrement de la coopération sociale sont les supports d'une mise en regard du stagiaire face à un modèle concret de travailleur employable (modèle incarné par ses pairs ou le formateur) qui sert de base à la construction de « troubles » de l'employabilité et à l'intériorisation de la norme; il s'agit du point de départ du travail d'intéressement pour associer le stagiaire à un projet d'employabilité. Les travailleurs en Entreprises de Formation par le Travail se positionnent donc dans le cadre de l'interaction. L'évaluation du stagiaire s'effectue dans des dispositifs de face-à-face enchevêtrés, elle se construit dans le cours de l'interaction; l'information produite est intersubjective. Le diagnostic d'employabilité se construit dans un long travail de mise en situation technique et sociale du stagiaire, qui peut servir de ressource pour un travail réflexif et interactif sur son employabilité, son projet, ses manques, ses moyens, ses carences. Loin d'être seulement fondé sur l'empathie relationnelle, ou sur des nomenclatures générales de métiers ou de formations, le diagnostic d'employabilité s'établit ici par l'interaction et la mise en situation dans un collectif organisé, une équipe, dont le stagiaire, disposé à se construire comme travailleur employable, est appelé à devenir un membre reconnu. Le dispositif est dès lors doublement fragile, par sa base interactionnelle tout d'abord, mais aussi par la longueur même du processus engagé.

Le cadre du réseau concernerait, à première vue, le conseiller en formation de Carrefour Formation. La construction (ou catégorisation) de «troubles de l'employabilité » s'opère au départ d'un travail de mise en relation. À la différence des CAP du FOREM, les règles administratives et les énoncés de la politique n'ont pas, à Carrefour, d'« effectivité automatique » sur le contenu du travail d'insertion. Ici, les espaces communs de travail, construits à la croisée de différentes organisations concurrentes oeuvrant dans un même champ, délimitent les contours de l'intervention du conseiller et en déterminent les modalités pratiques. Le travail en réseau consiste à mettre l'usager en relation avec les opérateurs de formation/insertion de la zone territoriale (dont le FOREM et les EFT). Cependant, le « réseau» ne s'étend pas aux entreprises: la construction de la norme d'employabilité s'opère, dès lors, en marge ou à distance du marché du travail. Le modèle du réseau doit donc être fortement nuancé au regard des limites relationnelles de l'agencement ; 
Carrefour Formation apparaît plutôt comme un compromis entre le modèle de l'institution (recours à des outils standards) et le modèle de l'interaction (intuition, empathie).

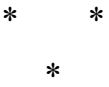

Ainsi, dans le champ de l'insertion socioprofessionnelle en Région wallonne, de nouveaux groupes professionnels proposent aux personnes sans emploi un traitement clinique du chômage. Il s'agit de diagnostiquer, chez les demandeurs d'emploi, des «troubles de l'employabilité », de les amener à « travailler sur eux-mêmes ", de les faire tendre vers une norme d'autonomie dans la vie sociale, en général, et la recherche d'un emploi, en particulier. Les politiques actives de l'emploi tendent ainsi à être tirées vers ce parti pris pour l' « humain », grâce au travail de groupes professionnels, dont la légitimité et la place dans la division du travail se construisent et se fondent sur l'expertise de la profession « consultante »- dont la caractéristique principale est la production de diagnostics par l'observation directe des symptômes -, à l'instar de la profession médicale analysée par Freidson.

Notre thèse est donc bien que conseillers en accompagnement professionnel, conseillers en formation, formateurs sociaux des EFT, sont, par-delà leurs différences, les vecteurs et les acteurs de la construction d'une nouvelle profession en gestation ${ }^{25}$, qui tend, ispo facto, à orienter les politiques publiques d'emploi dans le sens d'un traitement clinique ${ }^{26}$. Une telle incidence des professionnels de l'insertion sur la construction de l'action publique pourrait être discutée, en arguant que leur marge de manœuvre n'est pas si importante, dans la mesure où l'État tend très largement à cadrer leur autonomie ; en outre, ces groupes présentent malgré tout de fortes différences

\footnotetext{
${ }^{25}$ En effet, on est encore très loin d'un «marché du travail fermé », ou d'une " clôture » économique et sociale du segment professionnel des intermédiaires publics du marché du travail. Cependant, il n'existe pas de « point zéro » à la professionnalisation d'un métier.

${ }^{26}$ Rappelons que cette forme particulière et relativement inédite d'individualisation ou de responsabilisation des chômeurs ne se laisse nullement déduire de l'énoncé officiel des politiques actives d'emploi, tant au niveau européen ou fédéral que régional.
}

de pratiques, comme nous venons de l'illustrer. Nous voudrions relativiser ces arguments en guise de conclusion.

Malgré leurs différences, les trois groupes professionnels étudiés ont en commun d'être en rupture par rapport au "marché privé », à distance des intermédiaires privés du marché du travail et des dispositifs de mise en concurrence entre les candidats. Ils s'efforcent d'ailleurs de (re)construire - dans un contexte de gestion «mixte» du marché du travai127 - un monopole public sur le traitement clinique des chômeurs.

Cette définition commune du métier par la négative (contre les intermédiaires privés) n'est pas le seul élément qui atteste de la construction en cours d'une profession d'intermédiaire public en Région wallonne. Sur un plan identitaire, nous avons également relevé bon nombre de ritournelles ${ }^{28}$ professionnelles communes (Orianne, 2005b) qui délimitent des territoires de sens communs : «on n'est pas des flics de l'insertion ", "on n'est pas là pour parler en termes de solution », "on ne fait pas du quantitatif», etc. Les licences professionnelles (autorisations légales d'exercer) accordées par le Gouvernement wallon à ces différents groupes, ainsi que les mandats (traductions professionnelles des autorisations légales) produits au niveau de chaque organisation, témoignent également de ce même mouvement ou «devenir professionnel» du métier. Notons enfin que la segmentation entre généralistes et spécialistes de l'employabilité repose sur la construction d'interdépendances fonctionnelles entre groupes qui est aussi une condition de développement d'une identité professionnelle commune.

\footnotetext{
${ }^{27}$ Induit par la ratification, en Belgique, de la convention 181 de l'OIT (1997) autorisant les agences privées de placement.

${ }^{28} \mathrm{La}$ ritournelle est un énoncé (ou un ensemble d'énoncés) qui trace un territoire. Son caractère est répétitif et différencié ; sa fonction est territoriale. « Si la ritournelle territoriale passe si souvent dans les ritournelles professionnelles, c'est que les professions supposent que des activités fonctionnelles diverses s'exercent dans un même milieu, mais aussi que la même activité n'a pas d'autres agents dans le même territoire (...). Chez l'animal comme chez l'homme, ce sont les règles de distance critique pour l'exercice de la concurrence : mon coin de trottoir. Bref, il y a une territorialisation des fonctions qui est la condition de leur surgissement comme "travaux" ou "métiers" 》 (Deleuze et Guattari, 1980, p. 394).
} 
Ceci nous conduit à interroger les conditions de l'autonomie professionnelle de ces agents. Si tous les métiers luttent en principe pour obtenir un monopole sur l'exercice du travail qui est le leur, le droit d'exercer son autonomie, en toute légitimité, n'est reconnu qu'à la profession, nous dit Freidson (1984). Qu'en est-il des intermédiaires publics du marché du travail ? Certes, leur travail ne semble pas reposer sur un ensemble de qualifications et de connaissances hors du commun; en outre, les autorités publiques exercent un contrôle important sur l'organisation sociale et économique de leurs activités. Cependant, elles leur laissent simultanément le soin de définir le contenu de leur travail, ce qui est l'élément décisif de l'autonomie professionnelle.

L'intérêt de la démarche comparative, au cœur de notre étude, est d'avoir mis en évidence, par-delà les territoires organisationnels et professionnels du champ de l'insertion en Région wallonne, un nouveau paradigme de traitement du chômage : le traitement clinique. La dynamique, qui conduit ces différents groupes professionnels à infléchir (dans l'espace de la mise en œuvre et les limites de leur licence officielle) l'orientation des politiques d'emploi dans le sens d'un traitement clinique du chômage, nous apparaît comme une quête d'autonomie professionnelle (même si elle est précaire et potentiellement réversible): la recherche d'une « troisième voie» entre le tri du marché (opéré par les agences privées de placement) et la sanction de l'État fédéral (administrée par les «facilitateurs » de l'ONEM). Dans ce texte, le recours à la typologie d'Eymard-Duvernay et Marchal (la cartographie des régimes d'action des intermédiaires) donne à voir ce traitement idéal-typique, au travers de différentes pratiques et orientations qui se revendiquent toutes de ce même "parti pris réductionniste sur le modèle d'humanité » - pour reprendre l'expression de Robert Castel - au nom d'un ordre social qui ne passerait plus par l'imposition de contraintes mais par l'intériorisation de normes (Castel, 1981) ${ }^{29}$.

${ }^{29}$ Nous ne traiterons pas ici de l'intériorisation effective de ces normes (employabilité, adaptabilité, flexibilité, incitation à se former tout au long de la vie, etc.) ni de l'effet des différentes modalités de traitement clinique sur les trajectoires d'insertion des personnes. Nous renvoyons toutefois le lecteur à une typologie des usages du dispositif esquissée dans le cadre de cette recherche (Orianne et al., op. cit. : chapitre 6), à partir d'une trentaine d'entretiens d'usagers. Trois types d'usages ont pu ainsi être identifiés : l'usage proactif, l'usage réactif, et l'usage anomique.

\section{Bibliographie}

Alaluf M. (2004), «Le ministre, la morale et les chômeurs », Politique, avril 2004.

Barbier J.-C. et Gautié J. (1998) Les politiques d'emploi en Europe et aux États-Unis, Paris, Presses Universitaires de France.

Baszanger I. (1986), «Les maladies chroniques et leur ordre négocié », in Revue française de sociologie, XXVII, pp. 3-28.

Bessy C., Eymard-Duvernay F. (dir.) (1997), « Les intermédiaires du marché du travail», Cahiers $d u$ Centre d'Études de l'Emploi, $\mathrm{n}^{\circ}$ 36, PUF, Paris.
Bessy C., Eymard-Duvernay F., de Larquier G. et Marchal E. (dir.) (2001), Des marchés du travail équitables? Approche comparative France/RoyaumeUni, PIE-Peter Lang, Bruxelles.

Castel R. (1981), La gestion des risques. De l'antipsychiatrie à l'après-psychanalyse, Éditions de Minuit, Paris.

Castel R. (1995), Les métamorphoses de la question sociale. Une chronique du salariat, Fayard, Paris.

Deleuze G. \& Guattari F. (1980), Mille Plateaux, Capitalisme et schizophrénie 2, Éditions de Minuit, Paris. 
Dubar C. et Tripier P. (1998), Sociologie des professions, Armand Colin, Paris.

Dugué E. et Verger A. (2003), «Les inemployables », in Éducation Permanente, $\mathrm{n}^{\circ}$ 156/2003-3.

Duran P. (1999), Penser l'action publique, LGDJ, Paris.

Eymard-Duvernay F. et Marchal E. (1994), «Les règles en action: entre une organisation et ses usagers ", Revue française de sociologie, XXXV, pp. 5-36.

Eymard-Duvernay F. et Marchal E. (1997), Façons de recruter. Le jugement des compétences sur le marché du travail, Métaillé, Paris.

Faniel J. (2005), «Réactions syndicales et associatives face au contrôle de la disponibilité des chômeurs », L'année sociale 2004, pp. 133-148.

Foucault M. (1963), Naissance de la clinique, PUF, Paris.

Freidson E. (1984), La profession médicale, Payot, Paris.

Fusulier B. (1996), Entre exclusion et intégration: l'intervalle formateur. Étude des entreprises de formation par le travail, rapport de recherche pour la Fondation Roi Baudouin, Bruxelles.

Gautié J. (2003), «Quelle troisième voie? Repenser l'articulation entre marché du travail et protection sociale », Document de travail $n^{\circ} 30$, Centre d'Études de l'Emploi, Paris.

Gélot D. \& Nivolle P. (dir.) (2000), «Les intermédiaires des politiques publiques de l'emploi», Cahier Travail et Emploi, La Documentation française, Paris.

Goffman E. (1968), Asiles. Études sur la condition sociale des malades mentaux, Éditions de Minuit, Paris.

Hamzaoui M. (2005), « Émergence et logiques de la politique d'activation du social et de l'emploi», Pensée plurielle, 2005/2, n 10, pp. 19-28.
Hughes E.C. (1996), Le regard sociologique, Éditions de l'EHESS, Paris.

Maroy C. (2000), «Une typologie des référentiels d'action publique en matière de formation en Europe », in Recherches Sociologiques, 2000/2, Belgique, pp. 45-59.

Maroy C. et Van Haeperen B. (2005), « La structuration du champ de l'insertion en Wallonie: un processus inachevé », in Guyot J.-L., Mainguet C., Van Haeperen B. (Éds), La formation professionnelle continue. Enjeux sociétaux, De Boeck, Bruxelles, pp. 37-64.

Meyer J.-L. (1998), « Intermédiaires de l'emploi et marché du travail», Sociologie du travail, 3/98, pp. 345-364.

Orianne J.-F. (2005a), « L'État social actif en action : troubles de l'employabilité et traitement clinique du chômage », in Vielle P. et al., L'État social actif : vers un changement de paradigme?, PIE-Peter Lang, Bruxelles, pp. 179-207.

Orianne J.-F. (2005b), Le traitement clinique $d u$ chômage, Thèse de doctorat, UCL.

Orianne J.-F. (2006a), « Politiques actives d'emploi et professionnels de l'employabilité : critique et clinique », in Travail Emploi Formation, n 6/2006, Bruxelles, pp. 53-92.

Orianne J.-F. (2006b), « Carrefour formation : territorialisation de l'action publique et territoires professionnels », in Guyot J.-L. et Mainguet C. (dir.), La formation professionnelle continue. Stratégies collectives, De Boeck, «Économie Société Région », Bruxelles, pp. 263-303.

Orianne J.-F., Maroy C., Moulaert T., Vandenberghe V., Waltenberg F. (2004), Mises en ouvre locales des formules d'activation des politiques d'emploi, Academia Press, Gent.

Orianne J.-F. et Conter B. (2007), « Les politiques d'employabilité en Belgique : traitement clinique des chômeurs et traitement statistique du chômage », Recherches Sociologiques et Anthropologiques, vol. XXXVIII, $\mathrm{n}^{\circ}$ 2, pp. 175-190. 
Raveaud G. (2004a), Économie politique de la Stratégie Européenne pour l'Emploi, Thèse de doctorat en sciences économiques, université de Paris X Nanterre.

Simonin (dir.) (1995), «Les politiques publiques de l'emploi et leurs acteurs », Cahier du Centre d'Études de l'Emploi n ${ }^{\circ}$ 34, PUF, Paris.
Strauss A. (1992), La trame de la négociation. Sociologie qualitative et interactionnisme, textes réunis et présentés par Isabelle Baszanger, L'Harmattan, Paris.

Vrancken D. et Maquet C. (2006), Le travail sur soi. Vers une psychologisation de la société?, Belin, Paris.

\section{Résumé}

\section{Esquisse d'une profession consultante : les intermédiaires du marché du travail en Wallonie}

Jean-François Orianne et Christian Maroy

Cet article traite du rôle des intermédiaires publics du marché du travail dans l'orientation des politiques actives d'emploi en Belgique. Les auteurs présentent d'abord, de façon descriptive, le travail de ces agents à la lumière de la sociologie interactionniste des professions. Ils montrent ensuite que leur action paraît typique d'une profession consultante, contribuant à construire l'accompagnement des demandeurs d'emploi, en référence à une logique clinique, diagnostiquant et traitant des troubles de l'employabilité. Is soulignent enfin les variations que leur action présente au regard d'une typologie empruntée aux travaux conventionnalistes, et interrogent les conditions d'un devenir professionnel pour ces métiers émergeant des transformations de l'action publique ou, pour le dire autrement, de l'«activation» des politiques d'emploi.

Mots clés :

Métiers de l'emploi, métiers du conseil, politique de l'emploi, Belgique Journal of Economic Literature : J 28 